\title{
Pressure Dependence of Interatomic Separation and Thermal Expansivity for Alkali Halides and Periclase $(\mathrm{MgO})$
}

\author{
Q. LIU* \\ Department of Physics, Anhui University, Hefei 230039, China
}

(Received October 22, 2008; revised version February 3, 2009)

\begin{abstract}
In this paper, a new isothermal equation of state is developed based on an approximation for the volume dependence of the Anderson-Grüneisen parameter $\delta_{T}$ along isotherm. The values of interatomic separation $r$ with the change of pressure for nine alkali halides and periclase were investigated with the help of the new isotherm equation of state. The compression data are used to predict the pressure dependence of the coefficient of volume thermal expansion. The results are compared with the available experimental data and other theoretical results.
\end{abstract}

PACS numbers: 64.30.-t, 65.40.De, 91.60.Ki

\section{Introduction}

The isothermal equation of state (EOS) gives us valuable information about the change in volume under the influence of pressure at a fixed temperature and is thus related to the compression of solids. The theoretical studies of EOS at high pressure are of wide importance because they help us in planning future high pressure experiments and are also important in comparing static high pressure experiments with shock wave experiments. If we have a phenomenological form of EOS, we can predict the high-pressure properties and compression behavior of solids with a little effort by determining the parameters from theoretical calculations or experimental data. Various forms of the isothermal EOS [1-5] have been made to predict correctly high-pressure behaviors, irrespective of the material.

In this paper, we derive a new phenomenological EOS based on the approximation for the volume dependence of the Anderson-Grüneisen parameter $\delta_{T}$ along isotherm $[6,7]$. At the same time, we use it to calculate the values of interatomic separation $r$ with the change of pressure. The compression data obtained in the present work have also been used to calculate the pressure dependence of the volume thermal expansion coefficient. In order to examine the validity of presented isothermal EOS under study, we make a comparison of the calculated values and available experimental data as well as earlier theoretical investigations in the case of alkali halides and periclase.

The method of analysis is given in Sect. 2. The systematic comparison and discussion of the result are given in Sect. 3.

* e-mail: quanliu@ah172.com.cn

\section{Method of analysis}

Some useful thermodynamic relationships for predicting the pressure dependence of $r$ and $\alpha$ for solids can be obtained by the use of the definition of the AndersonGrüneisen parameter $\delta_{T}$ and with the help of certain physically plausible approximations which can be expressed as follows:

$$
\delta_{T}=-\frac{1}{\alpha B_{T}}\left(\frac{\mathrm{d} B_{T}}{\mathrm{~d} T}\right)_{P}
$$

where $\alpha$ and $B_{T}$ are the volume thermal expansion coefficient and the bulk modulus, respectively. These are defined as:

$$
\alpha=\frac{1}{V}\left(\frac{\mathrm{d} V}{\mathrm{~d} T}\right)_{P}
$$

and

$$
B_{T}=-V\left(\frac{\mathrm{d} P}{\mathrm{~d} V}\right)_{T} .
$$

The Maxwell thermodynamic relation is given as

$$
\left(\frac{\mathrm{d} \alpha}{\mathrm{d} P}\right)_{T}=\frac{1}{B_{T}^{2}}\left(\frac{\mathrm{d} B_{T}}{\mathrm{~d} T}\right)_{P} .
$$

Using Eqs. (1) and (2) and Eq. (4), we get

$$
\frac{\mathrm{d} \alpha}{\alpha}=\delta_{T} \frac{\mathrm{d} V}{V} .
$$

Integration of Eq. (5) gives the following result:

$$
\frac{\alpha}{\alpha_{0}}=\left(\frac{V}{V_{0}}\right)^{\delta_{T}} .
$$

Here $\alpha_{0}$ is the value of $\alpha$ at $V=V_{0}$. Now making use of the relation $V / V_{0}=\left(r / r_{0}\right)^{3}$, Eq. (6) can be rewritten as follows:

$$
\left(\frac{\alpha}{\alpha_{0}}\right)=\left(\frac{r}{r_{0}}\right)^{3 \delta_{T}} .
$$


It should be mentioned that Eq. (7) is based on the fact that $\delta_{T}$ is independent of $V$, i.e. of pressure and temperature.

On the other hand, it was show by Anderson et al. on the basis of investigations made by earlier worker that $\delta_{T}$ is not independent of $V$. Anderson et al. investigated the following relationship for the variation of $\delta_{T}$ along isotherms $[6,7]$ :

$$
\frac{\delta_{T}}{\delta_{T_{0}}}=\left(\frac{V}{V_{0}}\right)^{k},
$$

where $\delta_{T_{0}}$ is the value of $\delta_{T}$ at $V=V_{0}$ and $k$ is a dimensionless thermoelastic parameter whose value is about 1.5 $[6,7]$. It is interesting to mention here that for $k=1$, Eq. (8) reduces to the basic assumption considered in his formulation by Tallon [8]. In this paper, the value of $k$ is taken as 1.5 .

On the basis of an analysis of experimental data, Chopelas and Boehler ever investigated another relationship [9] for the isothermal variation of $\delta_{T}$ with volume

$$
\delta_{T}+1=\left(\delta_{T_{0}}+1\right) \frac{V}{V_{0}} .
$$

Although Eqs. (8) and (9) yield almost similar results, Eq. (8) was eventually chosen by Anderson et al. because of the convenience of the power law in the mathematical expressions inherent in thermodynamic identities.

If using Eq. (8) in Eq. (5), we obtained the following expression:

$$
\frac{\alpha}{\alpha_{0}}=\exp \left(-\frac{\delta_{T_{0}}}{k}\left(1-\eta^{k}\right)\right),
$$

where $\eta=V / V_{0}$, using the relation $\eta=V / V_{0}$, Eq. (10) can be modified as follows:

$$
\frac{\alpha}{\alpha_{0}}=\exp \left(-\frac{\delta_{T_{0}}}{k}\left(1-x^{3 k}\right)\right)
$$

where $x=r / r_{0}$. Then, substituting Eq. (10) into the most widely used approximation, $\alpha B_{T}=$ const [3]. We can obtain the following relationship between isothermal bulk modulus and relative volume compression:

$$
\frac{B_{T}}{B_{T_{0}}}=\exp \left(\frac{\delta_{T_{0}}}{k}\left(1-\eta^{k}\right)\right)
$$

where $B_{T_{0}}$ is the value of $B_{T}$ at $V=V_{0}$. Now taking Eq. (3) in Eq. (12), we get the following expression:

$$
\int_{V_{0}}^{V} \frac{1}{V} \exp \left(-\frac{\delta_{T_{0}}}{k}\left(1-\eta^{k}\right)\right) \mathrm{d} V=-\int_{0}^{P} \frac{\mathrm{d} P}{B_{T_{0}}} .
$$

The left hand side of above Eq. (13) cannot be integrated exactly. However, we can use an approximate method for evaluating this integral. Because in the range of pressure considered in this paper, the relative volume compression $V / V_{0}$ is not large, that means the value of $\left[\left(V / V_{0}\right)^{k}-1\right]$ is close to zero, and then the exponential function can be reduced to the following form:

$$
\begin{aligned}
& \int_{V_{0}}^{V} \frac{1}{V}\left[1+\frac{\delta_{T_{0}}}{k}\left(1-\eta^{k}\right)+\frac{\delta_{T_{0}}^{2}}{2 k^{2}}\left(1-\eta^{k}\right)^{2}\right] \mathrm{d} V= \\
& \quad-\frac{P}{B_{T_{0}}} .
\end{aligned}
$$

Integrating Eq. (14a), we get the following equation for volume compression:

$$
\begin{aligned}
& \left(1+\frac{\delta_{T_{0}}}{k}+\frac{\delta_{T_{0}}^{2}}{2 k^{2}}\right) \ln \eta+\frac{\delta_{T_{0}}}{k^{2}}\left(\frac{\delta_{T_{0}}}{k}+1\right)\left(1-\eta^{k}\right) \\
& -\frac{\delta_{T_{0}}^{2}}{4 k^{3}}\left(1-\eta^{2 k}\right)=-\frac{P}{B_{T_{0}}} .
\end{aligned}
$$

For studying the dependence of interatomic separation $r$ at room temperature, Eq. (14b) can be modified as follows with the help of $V / V_{0}=\left(r / r_{0}\right)^{3}$ :

$$
\begin{gathered}
\left(1+\frac{\delta_{T_{0}}}{k}+\frac{\delta_{T_{0}}^{2}}{2 k^{2}}\right) \ln x^{3}+\frac{\delta_{T_{0}}}{k^{2}}\left(\frac{\delta_{T_{0}}}{k}+1\right)\left(1-x^{3 k}\right) \\
-\frac{\delta_{T_{0}}^{2}}{4 k^{3}}\left(1-x^{6 k}\right)=-\frac{P}{B_{T_{0}}} .
\end{gathered}
$$

The above Eq. (11) and Eq. (14c) is the proper isotherm relationship for $\alpha$ and $\left(r / r_{0}\right)$ obtained in this paper. In order to test the validity of these expressions, we need to use Eq. (11) and (14c) to investigate the pressure dependence of $r$ and $\alpha$ for some solids.

\section{Result and discussion}

We have thus investigated the pressure-volume relationship using the methods based on the assumption for the variation of $\delta_{T}$ along isotherms $[6,7]$. The input data used in the calculations are given in Tables I and II. We have performed calculation for nine alkali halides and periclase up to a pressure of $45 \mathrm{kbar}$ because experimental measurements of pressure-volume data are limited. Vaidya and Kennedy [10] measured the volume of some halides up to $45 \mathrm{kbar}$ at room temperature using the piston-displacement method. Anderson and Zou [11] have reported the pressure-volume data for $\mathrm{MgO}$. These experimental results are included in Tables III-VI for the sake of comparison. From Tables III-VI, the results for $r$ vs. $P$ obtained in the present work are in good agreement with experimental data, supporting the validity of Anderson's assumption and Eq. (14c).

TABLE I

Values of input parameters used in the present work.

\begin{tabular}{c|c|c|c|c}
\hline \hline Crystal & $r_{0}[\AA][13]$ & $\alpha_{0}\left[10^{-4} k^{-1}\right][13]$ & $B_{T_{0}}[\mathrm{kbar}][13]$ & $\delta_{T_{0}}[14]$ \\
\hline $\mathrm{LiF}$ & 2.013 & 0.999 & 665 & 6.00 \\
$\mathrm{LiCl}$ & 2.570 & 1.32 & 297 & 6.77 \\
$\mathrm{LiBr}$ & 2.751 & 1.50 & 235 & 7.01 \\
$\mathrm{NaF}$ & 2.317 & 0.96 & 465 & 5.77 \\
$\mathrm{NaCl}$ & 2.820 & 1.19 & 240 & 5.85 \\
$\mathrm{NaBr}$ & 2.989 & 1.26 & 199 & 6.23
\end{tabular}

These calculated values of $r$ are then used to predict the pressure dependence of thermal expansion for each solid with the help of Eq. (11). The results of $\alpha$ as a function of pressure are reported in Tables III-VI. The experimental data for the $\alpha$ vs. $P$ are available only for $\mathrm{NaCl}$ [12] in the range of small compressions $0.9<\eta<1$. For convenience of comparison, the result given by Upadhyay 
TABLE II

Values of input parameters used in the present work.

\begin{tabular}{c|c|c|c|c}
\hline \hline Crystal & $r_{0}[\AA][15]$ & $\alpha_{0}\left[10^{-4} k^{-1}\right][15]$ & $B_{T_{0}}[\mathrm{kbar}][15]$ & $\delta_{T_{0}}[16]$ \\
\hline $\mathrm{CsCl}$ & 2.571 & 1.37 & 180 & 5.89 \\
$\mathrm{CsBr}$ & 2.720 & 1.39 & 159 & 5.71 \\
$\mathrm{CsI}$ & 3.956 & 1.46 & 128 & 5.79 \\
$\mathrm{MgO}$ & 2.105 & 0.312 & 173 & 4.29
\end{tabular}

and Kumar [17, 18] with Eq. (7) is also listed together with our calculated results. The values of $\alpha$ obtained in the present study as well as those reported by Kumar reveal that $\alpha$ decreases as the pressure is increased. However, the rate of variation of $\alpha$ with $P$ predicted from both studies differ appreciably from each other and this difference goes on increasing as the pressure is increased. At higher pressure the dependence of $\alpha$ with $P$ predicted in the present study is, in general, significantly more rapid than that reported by Kumar. We known that Kumar's result is based on the assumption $\delta_{T}$ is independent of pressure. But actually, this approximation is the simplest from recent works based on detailed and comprehensive analysis of experimental data $[19,20]$ which demonstrate that $\delta_{T}$ decreases with the falling of compression ratio. It is clear that for $\mathrm{NaCl}$ whose the experimental data $\alpha$ have been reported by Boehler and Kennedy [12], our calculating results are slightly better than Kumar's result, which further support the validity of Eq. (11).

TABLE III

Calculated values of $r$ (in $[\AA]$ ) and $\alpha$ (in $10^{-4} k^{-1}$ ) with the change of pressure for alkali halides $\mathrm{LiF}, \mathrm{LiCl}$, and $\mathrm{LiI}$.

\begin{tabular}{|c|c|c|c|c|c|c|c|c|c|c|c|c|}
\hline \multirow{3}{*}{$\begin{array}{c}P \\
{[\mathrm{kbar}]}\end{array}$} & \multicolumn{4}{|c|}{$\mathrm{LiF}$} & \multicolumn{4}{|c|}{$\mathrm{LiCl}$} & \multicolumn{4}{|c|}{ LiI } \\
\hline & \multicolumn{2}{|c|}{$r$} & \multicolumn{2}{|r|}{$\alpha$} & \multicolumn{2}{|c|}{$r$} & \multicolumn{2}{|r|}{$\alpha$} & \multicolumn{2}{|c|}{$r$} & \multicolumn{2}{|r|}{$\alpha$} \\
\hline & cal & [10] & $\mathrm{cal}$ & Kumar & cal & {$[10]$} & cal & Kumar & $\mathrm{cal}$ & [10] & cal & Kumar \\
\hline 0 & 2.013 & 2.013 & 0.999 & 0.999 & 2.570 & 2.570 & 1.32 & 1.32 & 2.751 & 2.751 & 1.50 & 1.50 \\
\hline 5 & 2.008 & 2.009 & 0.955 & 0.955 & 2.556 & 2.577 & 1.18 & 1.18 & 2.733 & 2.733 & 1.31 & 1.31 \\
\hline 10 & 2.003 & 2.004 & 0.914 & 0.913 & 2.544 & 2.545 & 1.08 & 1.07 & 2.717 & 2.717 & 1.17 & 1.15 \\
\hline 15 & 1.999 & 1.999 & 0.883 & 0.881 & 2.533 & 2.533 & 0.99 & 0.98 & 2.703 & 2.700 & 1.05 & 1.04 \\
\hline 20 & 1.995 & 1.994 & 0.853 & 0.849 & 2.523 & 2.522 & 0.92 & 0.91 & 2.688 & 2.685 & 0.96 & 0.94 \\
\hline 25 & 1.990 & 1.990 & 0.817 & 0.812 & 2.513 & 2.511 & 0.86 & 0.84 & 2.676 & 2.672 & 0.88 & 0.85 \\
\hline 30 & 1.986 & 1.986 & 0.789 & 0.783 & 2.504 & 2.501 & 0.80 & 0.78 & 2.662 & 2.658 & 0.82 & 0.78 \\
\hline 35 & 1.982 & 1.982 & 0.763 & 0.755 & 2.496 & 2.491 & 0.76 & 0.73 & 2.651 & 2.646 & 0.76 & 0.72 \\
\hline 40 & 1.979 & 1.978 & 0.744 & 0.735 & 2.488 & 2.481 & 0.72 & 0.68 & 2.637 & 2.634 & 0.71 & 0.67 \\
\hline 45 & 1.975 & 1.974 & 0.719 & 0.709 & 2.480 & 2.473 & 0.67 & 0.64 & 2.629 & 2.624 & 0.67 & 0.62 \\
\hline
\end{tabular}

TABLE IV

As Table III, for alkali halides $\mathrm{NaF}, \mathrm{NaCl}$, and $\mathrm{NaBr}$.

\begin{tabular}{|c|c|c|c|c|c|c|c|c|c|c|c|c|c|}
\hline \multirow{3}{*}{$\begin{array}{c}P \\
{[\mathrm{kbar}]}\end{array}$} & \multicolumn{4}{|c|}{$\mathrm{NaF}$} & \multicolumn{5}{|c|}{$\mathrm{NaCl}$} & \multicolumn{4}{|c|}{$\mathrm{NaBr}$} \\
\hline & \multicolumn{2}{|c|}{$r$} & \multicolumn{2}{|r|}{$\alpha$} & \multicolumn{2}{|c|}{$r$} & \multicolumn{3}{|c|}{$\alpha$} & \multicolumn{2}{|c|}{$r$} & \multicolumn{2}{|r|}{$\alpha$} \\
\hline & cal & [10] & cal & Kumar & cal & [10] & cal & Kumar & {$[12]$} & cal & [10] & cal & Kumar \\
\hline 0 & 2.317 & 2.317 & 0.96 & 0.96 & 2.820 & 2.820 & 1.19 & 1.19 & 1.19 & 2.989 & 2.989 & 1.26 & 1.26 \\
\hline 5 & 2.309 & 2.309 & 0.90 & 0.90 & 2.802 & 2.802 & 1.06 & 1.06 & 1.06 & 2.966 & 2.966 & 1.09 & 1.09 \\
\hline 10 & 2.301 & 2.301 & 0.85 & 0.85 & 2.785 & 2.786 & 0.96 & 0.96 & 0.98 & 2.946 & 2.945 & 0.97 & 0.96 \\
\hline 15 & 2.294 & 2.294 & 0.81 & 0.80 & 2.770 & 2.769 & 0.88 & 0.87 & 0.89 & 2.928 & 2.925 & 0.87 & 0.86 \\
\hline 20 & 2.287 & 2.287 & 0.77 & 0.76 & 2.757 & 2.755 & 0.82 & 0.80 & 0.83 & 2.911 & 2.908 & 0.79 & 0.77 \\
\hline 25 & 2.281 & 2.281 & 0.74 & 0.73 & 2.744 & 2.740 & 0.76 & 0.74 & 0.79 & 2.896 & 2.892 & 0.73 & 0.70 \\
\hline 30 & 2.275 & 2.275 & 0.71 & 0.69 & 2.732 & 2.729 & 0.71 & 0.68 & 0.73 & 2.883 & 2.877 & 0.67 & 0.64 \\
\hline 35 & 2.269 & 2.269 & 0.68 & 0.67 & 2.721 & 2.717 & 0.67 & 0.64 & & 2.870 & 2.863 & 0.63 & 0.59 \\
\hline 40 & 2.263 & 2.263 & 0.65 & 0.64 & 2.710 & 2.705 & 0.63 & 0.59 & & 2.856 & 2.849 & 0.59 & 0.55 \\
\hline 45 & 2.258 & 2.258 & 0.63 & 0.61 & 2.700 & 2.695 & 0.60 & 0.55 & & 2.845 & 2.837 & 0.56 & 0.51 \\
\hline
\end{tabular}


TABLE V

As Table III, for alkali halides $\mathrm{CsCl}, \mathrm{CsBr}$, and CsI.

\begin{tabular}{c|c|c|c|c|c|c|c|c|c|c|c|c}
\hline \hline \multirow{2}{*}{$\begin{array}{c}P \\
{[\mathrm{kbar}]}\end{array}$} & \multicolumn{4}{|c|}{ CsCl } & \multicolumn{4}{c|}{ CsBr } & \multicolumn{4}{c|}{ CsI } \\
\cline { 2 - 13 } & \multicolumn{2}{|c|}{$r$} & \multicolumn{2}{c|}{$\alpha$} & \multicolumn{2}{c|}{$r$} & \multicolumn{2}{c|}{$\alpha$} & \multicolumn{2}{|c|}{$r$} & \multicolumn{2}{c}{$\alpha$} \\
\cline { 2 - 12 } & cal & {$[10]$} & cal & Kumar & cal & {$[10]$} & cal & Kumar & cal & {$[10]$} & cal & Kumar \\
\hline 0 & 3.571 & 3.571 & 1.37 & 1.37 & 3.720 & 3.720 & 1.39 & 1.39 & 3.956 & 3.956 & 1.46 & 1.46 \\
10 & 3.514 & 3.511 & 1.04 & 1.03 & 3.654 & 3.650 & 1.03 & 1.02 & 3.871 & 3.870 & 1.02 & 1.00 \\
20 & 3.469 & 3.464 & 0.85 & 0.82 & 3.602 & 3.590 & 0.83 & 0.80 & 3.803 & 3.800 & 0.80 & 0.76 \\
30 & 3.432 & 3.425 & 0.72 & 0.68 & 3.560 & 3.550 & 0.70 & 0.65 & 3.755 & 3.750 & 0.65 & 0.60 \\
45 & 3.385 & 3.378 & 0.59 & 0.53 & 3.507 & 3.500 & 0.57 & 0.51 & 3.690 & 3.682 & 0.53 & 0.45
\end{tabular}

TABLE VI

As Table III, for periclase $(\mathrm{MgO})$.

\begin{tabular}{c|c|c|c|c}
\hline \hline \multirow{2}{*}{$\begin{array}{c}P \\
{[\mathrm{kbar}]}\end{array}$} & \multicolumn{4}{|c}{$\mathrm{MgO}$} \\
\cline { 2 - 5 } & \multicolumn{2}{|c|}{$r$} & \multicolumn{2}{c}{$\alpha$} \\
\cline { 2 - 5 } & cal & {$[11]$} & cal & Kumar \\
\hline 0 & 2.105 & 2.105 & 0.312 & 0.312 \\
3.52 & 2.091 & 2.091 & 0.287 & 0.286 \\
7.54 & 2.077 & 2.077 & 0.264 & 0.263 \\
12.00 & 2.063 & 2.062 & 0.243 & 0.241 \\
16.25 & 2.050 & 2.047 & 0.226 & 0.222 \\
21.88 & 2.034 & 2.032 & 0.207 & 0.201 \\
27.45 & 2.020 & 2.017 & 0.192 & 0.184 \\
34.25 & 2.004 & 2.002 & 0.177 & 0.166 \\
41.55 & 1.989 & 1.986 & 0.164 & 0.150 \\
58.55 & 1.956 & 1.954 & 0.140 & 0.121 \\
81.56 & 1.919 & 1.921 & 0.118 & 0.094 \\
109.00 & 1.884 & 1.887 & 0.100 & 0.073
\end{tabular}

To summarize, the assumption $[6,7]$ for the variation of $\delta_{T}$ along isotherms leads to simple relation for determining the pressure dependence of interatomic separation and thermal expansion coefficients. The simplicity of the present method makes it possible to apply the formulae for studying more complex solids like minerals of geophysical importance and application.

\section{Acknowledgments}

This work was financially supported by Key Laboratory of Opto-electronic Information Acquisition and Manipulation of Ministry of Education of China (No. 9603) and by the Natural Science Foundation of the Education Department of Anhui Province (No. KJ2008B014).

\section{References}

[1] F.D. Murnaghan, Proc. Natl. Acad. Sci. 30, 244 (1944).

[2] P. Vinet, J. Ferrante, J.H. Rose, J.R. Smith, J. Geophys. Res. 92, 9319 (1987).

[3] M. Kumar, Physica B 212, 341 (1995); 217, 143 (1995).

[4] P.B. Roy, S.B. Roy, Physica B 350, 375 (2004).

[5] S.S. Kushwah, Phys. Earth Planet. Int. 149, 201 (2005).

[6] O.L. Anderson, H. Oda, D.G. Issak, Geophys. Res. Lett. 19, 1987 (1992).

[7] O.L. Anderson, D.G. Issak, J. Phys. Chem. Solids 54, 221 (1993).

[8] J.L. Tallon, J. Phys. Chem. Solids 41, 837 (1980).

[9] A. Chopelas, R. Boehler, Geophys. Res. Lett. 19, 1083 (1992).

[10] S.N. Vaidya, G.C. Kennedy, J. Phys. Chem. Solids 32, 951 (1971).

[11] O.L. Anderson, K. Zou, J. Phys. Chem. Ref. Data 19, 69 (1990).

[12] R. Boehler, G.C. Kennedy, J. Phys. Chem. Solids 41, 517, 1019 (1980).

[13] A. Dhoble, M.P. Verma, Phys. Status Solidi B 136, 497 (1986).

[14] J. Shanker, K. Singh, J. Inorg. Nucl. Chem. 43, 1445 (1981).

[15] R.M. Hazin, L.W. Finger, Comparative Crystal Chemistry, Wiley, Chichester 1982.

[16] M.P. Tost, Solid. State Phys. 16, 1 (1964).

[17] S.P. Upadhyay, M. Kumar, Phys. Status Solidi B 191, 299 (1995).

[18] M. Kumar, S.P. Upadhyay, J. Phys. Chem. Solids 54, 773 (1993).

[19] O.L. Anderson, Equation of State of Solids for Geophysics and Ceramic Science, Oxford University Press, Oxford 1995.

[20] D.G. Issak, O.L. Anderson, Physica B 328, 345 (2003). 